\title{
The route to magnetic order in the spin- $1 / 2$ kagome Heisenberg antiferromagnet: The role of interlayer coupling
}

\author{
O. Götze and J. Richter \\ Institut für Theoretische Physik, Universität Magdeburg, 39016 Magdeburg, Germany
}

\begin{abstract}
While the existence of a spin-liquid ground state of the spin-1/2 kagome Heisenberg antiferromagnet (KHAF) is well established, the discussion of the effect of an interlayer coupling (ILC) by controlled theoretical approaches is still lacking. Here we study this problem by using the coupledcluster method to high orders of approximation. We consider a stacked KHAF with a perpendicular ILC $J_{\perp}$, where we study ferro- as well as antiferromagnetic $J_{\perp}$. We find that the spin-liquid ground state (GS) persists until relatively large strengths of the ILC. Only if the strength of the ILC exceeds about $15 \%$ of the intralayer coupling the spin-liquid phase gives way for $q=0$ magnetic long-range order, where the transition between both phases is continuous and the critical strength of the ILC, $\left|J_{\perp}^{c}\right|$, is almost independent of the sign of $J_{\perp}$. Thus, by contrast to the quantum GS selection of the strictly two-dimensional KHAF at large spin $s$, the ILC leads first to a selection of the $q=0$ GS. Only at larger $\left|J_{\perp}\right|$ the ILC drives a first-order transition to the $\sqrt{3} \times \sqrt{3}$ long-range ordered GS. As a result, the stacked spin-1/2 KHAF exhibits a rich GS phase diagram with two continuous and two discontinuous transitions driven by the ILC.

PACS numbers: 75.10.Jm, 75.10.Kt, 75.50.Ee, $75.45 .+\mathrm{j}$
\end{abstract}

Introduction.- The search for exotic quantum spin liquid (QSL) states and fractionalized quasiparticles in frustrated magnets attracts currently much attention both from the theoretical and experimental side. One of the most promising, fascinating, and, at same time, challenging problems is the investigation of the ground state (GS) of the quantum antiferromagnet on the kagome lattice. Over the last 25 years a plethora of theoretical approaches has been applied to understand the GS properties of the spin-1/2 kagome antiferromagnet (KAFM), see, e.g., Refs. [1 18]. Clearly, the GS of the $s=1 / 2$ Heisenberg KAFM does not exhibit GS magnetic longrange order (LRO). However, there is a long-standing debate on the nature of the quantum GS. Recent largescale numerical studies [6, 8, 11] provide arguments for a gapped $\mathbb{Z}_{2}$ topological QSL for spin $s=1 / 2$. However, the gap state is not fully proven, and also a gapless spin liquid is suggested, see, e.g., Refs. [9, 12, 16].

A natural question is that for the stability of the QSL phase against modifications of the paradigmatic pure $s=1 / 2$ KAFM. Several recent investigations have been focused on $s>1 / 2$ [10, 19 25], anisotropic models [16, 25 34] as well as KAFMs with further-neighbor couplings 31, 35 48]. It has been found that such modifications of the pure KAFM may play a crucial role either to modify the QSL state or even to establish GS magnetic LRO of $\sqrt{3} \times \sqrt{3}$ or of $q=0$ symmetry. At that the input from experiments plays an important role to trigger the theoretical hunt for exotic quantum states [48 64]. Prominent examples for $s=1 / 2$ kagome compounds are herbertsmithite [49 53] and kapellasite [54, 55]. Both compounds do not show magnetic order down to very low temperatures [49 55]. However, the underlying magnetic model is quite different. Herbertsmithite is likely the best realization of a spin- $1 / 2$ Heisenberg KAFM with only nearest-neighbor (NN) exchange couplings. On the other hand, the model for kapellasite contains noticeable further-neighbor couplings $J_{d}$ along the diagonals of the hexagons [36, 48, 56, 57]. Except the kagome compounds without magnetic order there are several kagome magnets which exhibit a phase transition to a long-range ordered state at a critical temperature $T_{c}$. Examples are edwardsite [58], barlowite [59, 60] or the family of kagome compounds $\mathrm{Cs}_{2} \mathrm{Cu}_{3} \mathrm{MF}_{12}(\mathrm{M}=\mathrm{Zr}, \mathrm{Hf}, \mathrm{Sn}) 61$ 63. For an overview on the relation between extended models and kagome compounds we refer the interested reader to Ref. [48].

Bearing in mind the huge number of theoretical studies of purely two-dimensional (2D) kagome models, see, e.g., Refs. [1 48], the investigation of the role of interlayer coupling (ILC) $J_{i l}$ so far has been widely ignored. The reason for that might be related to the fact that most of the controlled approaches with satisfactory accuracy, such as large-scale exact diagonalization, density matrix renormalization group (DMRG), or entanglement renormalization techniques are designed for low-dimensional quantum systems. Thus, for example, for the three-dimensional (3D) counterpart of the KAFM, the quantum pyrochlore Heisenberg antiferromagnet (HAFM), precise GS data are missing so far. To the best of our knowledge the stacked kagome spin-1/2 HAFM was studied only in an early paper by using a rotational invariant Green's function approach [65]. Certainly, one can expect that in kagome compounds an ILC is present. The geometry and the strength of $J_{i l}$ may differ from compound to compound. Unquestionably, an ILC is crucial to establish magnetic LRO at finite temperatures, at least if the spin anisotropy is negligible.

In the present paper we study the spin- $1 / 2$ HAFM on 
the stacked kagome lattice described by

$$
H=\sum_{n}\left(\sum_{\langle i j\rangle} \mathbf{s}_{i, n} \cdot \mathbf{s}_{j, n}\right)+J_{\perp} \sum_{i, n} \mathbf{s}_{i, n} \cdot \mathbf{s}_{i, n+1},
$$

where $n$ labels the kagome layers and $J_{\perp}$ is a perpendicular (i.e. non-frustrated) ILC. The expression in brackets represents the kagome HAFM model of the layer $n$ with $\mathrm{NN}$ intralayer couplings $J=1$. For $J_{\perp}$ we consider antiferromagnetic (AFM) as well as ferromagnetic (FM) couplings.

The questions we want to address in the present paper are as follows: Is the perpendicular ILC $J_{\perp}$ able to establish magnetic LRO for kagome $s=1 / 2$ layers with AFM isotropic NN interactions, at all? As we will demonstrate below the answer is 'yes'. Then, as consequent questions arise: Does the magnetically disordered GS survive a (small) finite (non-frustrated) ILC? Which GS magnetic LRO (i.e. $\sqrt{3} \times \sqrt{3}$ or $q=0$ ) is selected? Is the sign of $J_{\perp}$ relevant? If for $\left|J_{\perp}\right|>0$ GS magnetic LRO is present, we may expect that for the $3 \mathrm{D}$ system at hand a finite critical temperature $T_{c}$ exists. From previous studies of coupled low-dimensional Heisenberg spin systems [66, 67] we know that $T_{c}$ may grow as a logarithmic function of $J_{\perp}$ slightly beyond the quantum phase transition to GS magnetic LRO.

In order to address the above asked questions concerning the role of the ILC we use the coupled cluster method (CCM) 68, 69] to high orders of approximation. The CCM is a very general $a b$ initio many-body technique that has been successfully applied to strongly frustrated quantum magnets [10, 30, 37, 39, 70 80]. The precision of the method has been demonstrated for kagome spin systems in Refs. 10] and 30]. Thus, the CCM GS energy for the $s=1 / 2$ isotropic Heisenberg KAFM is close to best available DMRG results [6, 11]. By contrast to exact diagonalization, DMRG, or entanglement renormalization techniques the CCM can be applied straightforwardly to 3D systems [70, 81].

Coupled cluster method (CCM).- We illustrate here only some basic relevant features of the CCM. At that we follow Refs. [10] and [30], where the CCM was applied to the 2D KAFM. For more general information on the CCM, see, Refs. 69, 81 84]. Note first that the CCM yields results directly for number of sites $N \rightarrow \infty$. As a starting point of the CCM calculation we choose a normalized reference state $|\Phi\rangle$. From a quasi-classical point of view that is for the system at hand the stacked coplanar $\sqrt{3} \times \sqrt{3}$ or $q=0$ state (see, e.g., Refs. [28, 30, 8588]). We perform a rotation of the local axes of each of the spins such that all spins in the reference state align along the negative $z$ axis. Within the framework of the local spin coordinates we define a complete set of multispin creation operators $C_{I}^{+} \equiv\left(C_{I}^{-}\right)^{\dagger}$ related to this reference state: $|\Phi\rangle=|\downarrow \downarrow \downarrow \cdots\rangle ; C_{I}^{+}=$ $s_{n}^{+}, s_{n}^{+} s_{m}^{+}, s_{n}^{+} s_{m}^{+} s_{k}^{+}, \ldots$, . Here the spin operators are defined in the local rotated coordinate frames. The indices $n, m, k, \ldots$ denote arbitrary lattice sites. The ket and bra GS eigenvectors $|\Psi\rangle$ and $\langle\tilde{\Psi}|$ of the spin system are given by $|\Psi\rangle=e^{S}|\Phi\rangle, S=\sum_{I \neq 0} a_{I} C_{I}^{+}$; $\langle\tilde{\Psi}|=\langle\Phi| \tilde{S} e^{-S}, \tilde{S}=1+\sum_{I \neq 0} \tilde{a}_{I} C_{I}^{-}$. The coefficients $a_{I}$ and $\tilde{a}_{I}$ in the CCM correlation operators, $S$ and $\tilde{S}$, can be determined by the ket-state and bra-state equations $\left\langle\Phi\left|C_{I}^{-} e^{-S} H e^{S}\right| \Phi\right\rangle=0 ;\left\langle\Phi\left|\tilde{S} e^{-S}\left[H, C_{I}^{+}\right] e^{S}\right| \Phi\right\rangle=$ $0 ; \forall I \neq 0$. Each equation belongs to a certain configuration index $I$, i.e., it corresponds to a certain configuration of lattice sites $n, m, k, \ldots$. From the Schrödinger equation, $H|\Psi\rangle=E_{0}|\Psi\rangle$, we get for the GS energy $E_{0}=$ $\left\langle\Phi\left|e^{-S} H e^{S}\right| \Phi\right\rangle$. The magnetic order parameter (sublattice magnetization) is given by $M=-\frac{1}{N} \sum_{i=1}^{N}\left\langle\tilde{\Psi}\left|s_{i}^{z}\right| \Psi\right\rangle$, where $s_{i}^{z}$ is expressed in the transformed coordinate system. For the solution of the ket-state and bra-state equations we use the well established LSUB $m$ approximation scheme, in order to truncate the expansions of $S$ and $\tilde{S}$, cf., e.g., Refs. [10, 30, 37, 39, 70 75, 77 80, 82, 83]. In the LSUB $m$ scheme no more than $m$ spin flips spanning a range of no more than $m$ contiguous lattice sites are included. Using an efficient parallelized CCM code [89] we can solve the CCM equations up to LSUB8 for $s=1 / 2$. Following Refs. [10, 30] we extrapolate the 'raw' LSUB $m$ data to the limit $m \rightarrow \infty$. Here we use two schemes, namely an extrapolation using $m=4,5, \ldots, 8$ (scheme I) and separetely an extrapolation using $m=4,6,8$ (scheme II). The former one corresponds to that used for the $2 \mathrm{D}$ KAFM [10, 30], whereas scheme II (i.e., omitting the odd LSUB $m$ approximation levels) is more appropriate for magnets with collinear AFM correlations 69 72, 7476, 79]. By comparing the results of both schemes we can get an idea on the precision of the extrapolated data.

For the GS energy the ansatz $e_{0}(m)=E_{0}(m) / N=$ $e_{0}(m \rightarrow \infty)+a_{1} / m^{2}+a_{2} / m^{4}$ provides accurate data for the extrapolated energy $e_{0}(m \rightarrow \infty)$, whereas for the magnetic order parameter $M$ the ansatz $M(m)=$ $M(m \rightarrow \infty)+b_{1}(1 / m)^{x}+b_{2}(1 / m)^{x+1}$ is appropriate. The choice of the leading exponent $x$ is a subtle issue, since $x$ might be different in semi-classical GS phases with wellpronounced magnetic LRO and near a quantum critical point, see [10, 30, 37, 39, 70-72, 74, 75, 79, 80]. For the kagome problem at hand we start from a magnetically disordered phase at $J_{\perp}=0$ and search for quantum phase transitions to GSs with magnetic LRO. For that the extrapolation of $M$ with $x=1 / 2$ is the best choice as it has been demonstrated in many previous CCM investigations [10, 30, 37, 39, 71, 74, 75, 79, 80]. Thus, the CCM treatment of the celebrated spin-half $J_{1}-J_{2}$ model on the square lattice using $x=1 / 2[71,79]$ yields quantum critical points, which are in very good agreement with best available numerical results obtained by DMRG with explicit implementation of $\mathrm{SU}(2)$ spin rotation symmetry [90].

Results and Discussion.- We start with a brief discus- 
sion of the GS energy per spin $e_{0}=E_{0} / N$, shown in the insets of Figs. 1 (a) and (b) for the $\sqrt{3} \times \sqrt{3}$ and $q=0$ reference states, respectively. We see that $e_{0}$ converges quickly as the level $m$ of the LSUB $m$ approximation increases. Hence, the extrapolation with leading order $1 / \mathrm{m}^{2}$ can be considered as very accurate, as it has been demonstrated in many cases, where data from other precise methods are available to compare with, see, e.g. Refs. 10, 30, 69]. Moreover, the results of both extrapolation schemes are almost indistinguishable. The shape of the curves and the magnitude of the energies is very similar for both states. From Ref. [10] we know that at $J_{\perp}=0$ the $q=0$ state has slightly lower energy. The extrapolated GS energy behaves smoothly as changing the sign of $J_{\perp}$. The magnetic order parameter $M$ for the $\sqrt{3} \times \sqrt{3}$ and $q=0$ states is shown in the main panel of Figs. 1(a) and (b). Of course, $M$ is zero for $J_{\perp}=0[10,30]$. As a main result, we find that the ILC is able to establish magnetic LRO for kagome $s=1 / 2$ layers with AFM NN Heisenberg interactions. The critical ILCs, where magnetic LRO sets in, are (i) $J_{\perp}=-0.100, J_{\perp}=+0.102(\sqrt{3} \times \sqrt{3}$ state $)$ and $J_{\perp}=-0.154, J_{\perp}=+0.151$ ( $q=0$ state $)$ for scheme I, and (ii) $J_{\perp}=-0.104, J_{\perp}=+0.110(\sqrt{3} \times \sqrt{3}$ state $)$ and $J_{\perp}=-0.135, J_{\perp}=+0.130$ ( $q=0$ state $)$ for scheme II. Thus, there is a reasonable agreement of the critical ILCs obtained by both extrapolation schemes. We notice that the amount of the critical $\left|J_{\perp}\right|$ is of comparable size as the spin gap estimated, e.g., in Refs. [3] and [11]. We may also compare with the square-lattice $J_{1}-J_{2}$ HAFM in the limit of strong frustration, i.e., at $J_{2} / J_{1} \sim 0.5$. The critical ILC $J_{\perp}$ found by various approaches $[70,91,92$ ] is $J_{\perp} \approx 0.12-0.2 J_{1}$, i.e., its size is comparable to that reported here for the kagome system. The behavior of $M$ near the critical $J_{\perp}$ indicates a typical second-order transition, where the slope of $M$ is quite steep. On the FM side $\left(J_{\perp}<0\right)$ there is a monotonic increase of $M$ with increasing $\left|J_{\perp}\right|$, and, both schemes I and II lead to very similar $M\left(J_{\perp}\right)$ curves. By contrast, on the AFM side $\left(J_{\perp}>0\right)$ there is a noticeable difference between both schemes. That can be attributed to emerging collinear AFM correlations along the AFM $J_{\perp}$ bonds that may lead to a different scaling of odd and even LSUB $m$ data [93]. We mention, that the maximum value of $M$ remains small even at $J_{\perp} \sim 1$. Note that for $\mathrm{AFM} J_{\perp}$ we have calculated data up to $J_{\perp}=100$. For the extrapolation scheme II relevant in the limit of large $J_{\perp}$ we do not find indications for a breakdown of LRO at a finite $J_{\perp}$, rather there is a monotonic decrease of $M$ with increasing $J_{\perp}$ reaching adiabatically $M=0$ at infinite $J_{\perp}$, cf. also Ref. [72].

Next we discuss the question which magnetic LRO is selected by quantum fluctuations. As it has been very recently demonstrated [28, 30] the mechanism of quantum selection of the GS LRO in the KAFM is very subtle and it is related to topologically nontrivial, looplike
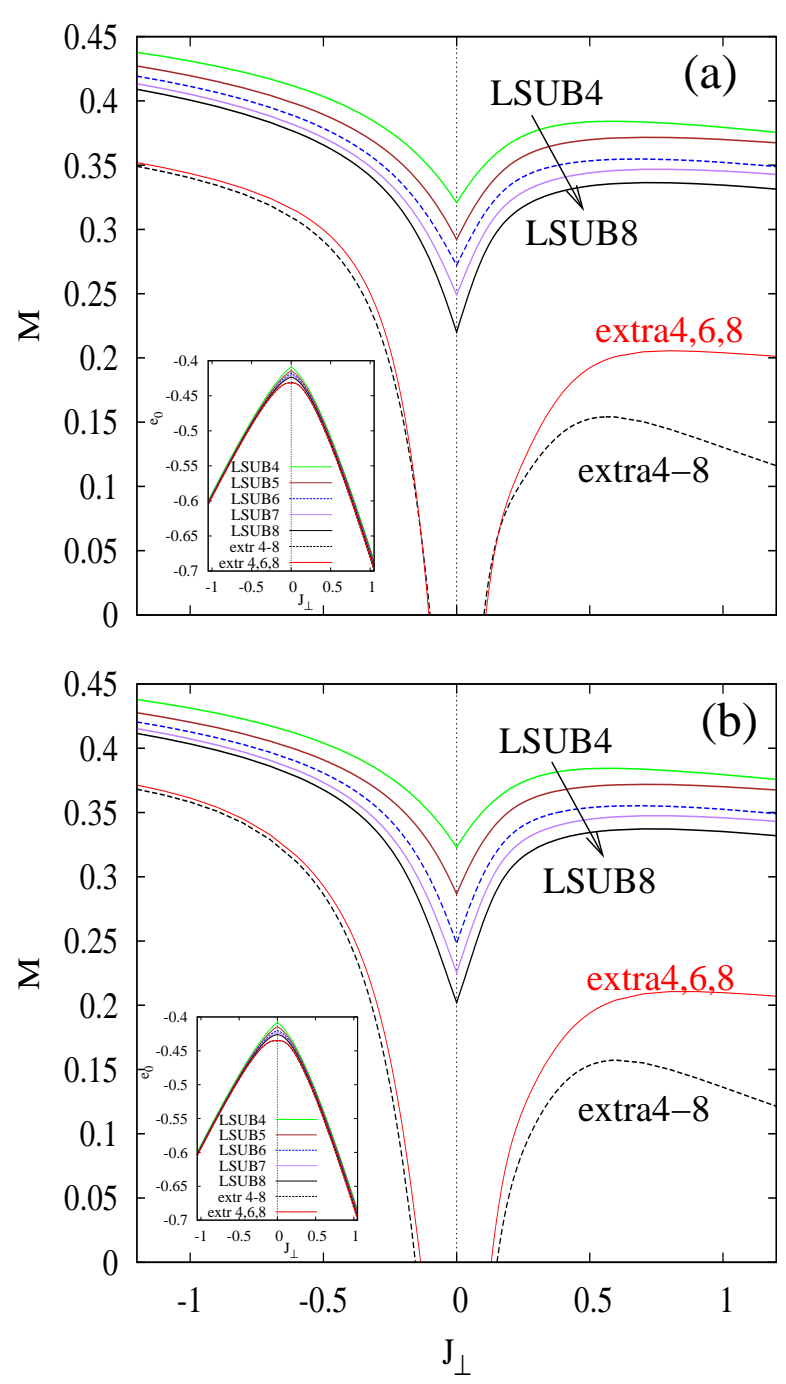

Figure 1. CCM-LSUBm as well as extrapolated GS sublattice magnetization $M$ using the $\sqrt{3} \times \sqrt{3}$ reference state (a) and the $q=0$ reference state (b) as a function of the ILC $J_{\perp}$. The labels 'extra4-8' and 'extra4,6,8' correspond to the extrapolation schemes I and II, respectively (see main text). Insets: Corresponding data for the GS energy $e_{0}$.

high-order spin-flip processes [28]. As a result, the energy difference between competing states is very small, e.g., about $10^{-4} J$ for the $X X Z$-KAFM [28, 30]. Hence, it is crucial to have a theory at hand that provides very accurate results for the GS energy and is able to take into account such high-order spin-flip processes. These criteria are fulfilled by the CCM, if high orders of approximation are considered. Thus, the quantum selection of the $\sqrt{3} \times \sqrt{3}$ GS vs. the $q=0$ GS obtained by non-linear spin-wave theory is also obtained by CCM for $s>1 / 2$ [10]. Very recently, a direct comparison of CCM and nonlinear spin-wave data for energy differences (which are 


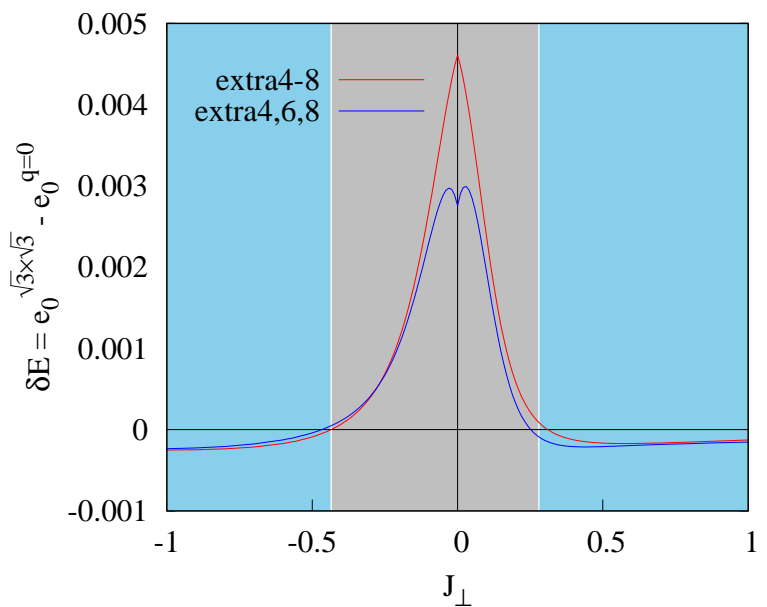

Figure 2. Difference $\delta e=e_{0}^{\sqrt{3} \times \sqrt{3}}-e_{0}^{q=0}$ of the extrapolated GS energies of the $\sqrt{3} \times \sqrt{3}$ and the $q=0$ states as a function of the ILC $J_{\perp}$. The labels 'extra4-8' and 'extra4,6,8' correspond to the extrapolation schemes I and II, respectively (see main text).

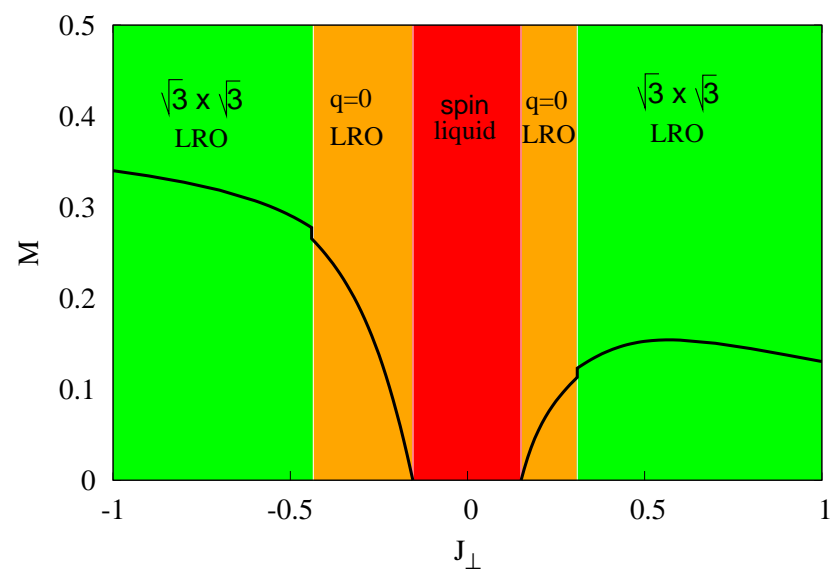

Figure 3. Sketch of the GS phases of the stacked spin-1/2 Heisenberg KAFM. The black curve shows the magnetic order parameter $M$ in the GS phases with magnetic LRO using extrapolation scheme I.

also of the order of few $10^{-3}$ ) for the $X X Z$ KAFM for large $s$ has been given, see Fig. 3 in Ref. [30], which provides evidence that both independent approaches agree very well. Thus, we may conclude that our results for the quantum selection are trustworthy. We show our results for the energy difference $\delta e=e_{0}^{\sqrt{3} \times \sqrt{3}}-e_{0}^{q=0}$ as a function of $J_{\perp}$ in Fig. 2. We mention first that both extrapolation schemes I and II yield consistent results for $\delta e$. At low values of $\left|J_{\perp}\right|$ the $q=0$ reference state yields lower energy, i.e. $\delta e>0$. That is in accordance with
Refs. [10] and [30], where the case $J_{\perp}=0$ was considered. On both sides $\delta e$ is still positive at those values of $J_{\perp}$, where the sublattice magnetizations $M_{\sqrt{3} \times \sqrt{3}}$ or $M_{q=0}$ become larger than zero. Hence, our results provide evidence that there is a magnetic disorder-to-order transition to $q=0 \mathrm{LRO}$ at $J_{\perp} \sim-(0.14 \ldots 0.15)$ and $J_{\perp} \sim+(0.13 \ldots 0.15)$, respectively, where this transition is likely continuous. Note that the quantum selection of the $q=0$ GS LRO is contrary to the semi-classical large- $s$ order-by-disorder selection of the $\sqrt{3} \times \sqrt{3}$ LRO found for the 2D spin-s KAFM. Further increasing the strength of $J_{\perp}$ leads to a second transition from $q=0$ to $\sqrt{3} \times \sqrt{3}$ LRO on the FM side at $J_{\perp}=-0.435$ (scheme I) and $J_{\perp}=-0.467$ (scheme II). At the AFM side we find $J_{\perp}=0.310$ (scheme I) and $J_{\perp}=0.252$ (scheme II). By contrast to the first transition this second transition is a discontinuous one between two ordered GS phases with different symmetries. On the FM side we may understand the realization of $\sqrt{3} \times \sqrt{3}$ LRO in terms of the large- $s$ order-by-disorder GS selection of the $\sqrt{3} \times \sqrt{3}$ state. Increasing the strength of the FM ILC leads to an effective composite spin with higher spin quantum number. However, this kind of mechanism does not work for AFM $J_{\perp}$, and to clarify the mechanism responsible for changing the GS selection remains an open question.

Collecting our results we obtain a sketch of the GS phase diagram of the stacked spin- $1 / 2$ Heisenberg KAFM as shown in Fig. 3. The system exhibits four transitions, two continuous ones between a QSL state and a magnetically ordered state with $q=0$ symmetry at $J_{\perp} \sim-(0.14 \ldots 0.15)$ and $J_{\perp} \sim+(0.13 \ldots 0.15)$, and two discontinuous ones between states with magnetic LRO of $q=0$ and $\sqrt{3} \times \sqrt{3}$ symmetry at $J_{\perp} \sim-(0.44 \ldots 0.47)$ and $J_{\perp} \sim+(0.25 \ldots 0.31)$. We further argue, that this kind of phase diagram is specific for the extreme quantum case $s=1 / 2$. From Ref. [10] we know that already for $s=1$ (and also for $s>1$ ) the $\sqrt{3} \times \sqrt{3}$ reference state has the lower energy. It seems to be very unlikely that this preference of the $\sqrt{3} \times \sqrt{3}$ state is changed by $J_{\perp}$.

Concluding remarks. Let us discuss the relation of our findings to the previous results based on a rotational invariant Green's function method (RGM) 65, where the existence of a non-magnetic GS for arbitrary values of $J_{\perp}$ was reported. To evaluate this discrepancy we have to assess the accuracy of the current CCM approach and of the RGM approach. First we mention that the CCM is a systematic approach taking into account all spin-flip processes up to a well-defined order. On the other hand, the decoupling of the equation of motion used in the RGM contains uncontrolled elements of approximation. Meanwhile, there is ample of experience in applying the RGM on frustrated quantum magnets, see, e.g., Ref. [96] and references therein. In its minimal version (used in Ref. 65]), where as many vertex parameters are used as independent conditions for them can be formulated, the accuracy of the description of GS properties seems to be 
limited [70, 94, 95]. In particular, the rotational invariant decoupling strongly overestimates the region of QSL phases. Thus, for the square-lattice $s=1 / 2 J_{1}-J_{2}$ HAFM the minimal version of the RGM predicts a QSL phase in an extremely wide region $0.1 \lesssim J_{2} / J_{1} \lesssim 1.7$, cf., e.g., Refs. [94, 95], instead of $0.44 \lesssim J_{2} / J_{1} \lesssim 0.6$, obtained by recent DMRG calculations [90] and also by the CCM [71, 79]. Another indication is the fairly poor GS energy of $e_{0}=-0.4296$ 65, 97], that is more than $2 \%$ above the best available DMRG energy $e_{0}=-0.4386$. (Note that the CCM energy obtained in Ref. [10] is $e_{0}=-0.4372$.) Thus we have evidence that the CCM description of the GS properties is much more reliable than the RGM in its minimal version.

Let us finally discuss the relevance of our results for experiments on kagome compounds. In real kagome compounds typically the interlayer coupling is more sophisticated than that we consider in our paper. Thus, there is only an indirect relation of our results to those compounds, which concerns the general question for the crossover from a purely $2 \mathrm{D}$ to a quasi-2D and finally to a three-dimensional system. However, there is at least one example with stacked (unshifted) kagome layers, namely barlowite. As it has been pointed out very recently, through isoelectronic substitution in barlowite this kagome system fits to our model system [99].

A main finding of our paper is that the QSL phase can be observed even if there is a sizeable ILC. Therefore, in accordance with the experimental observation the ILC of about $5 \%$ of the intralayer coupling as reported for herbertsmithite 98] and the ILC of about 6-7\% predicted for the modified barlowite system [99] is not sufficient to destroy the QSL phase. On the other hand, if the ILC is large enough (about 15\% of the intralayer coupling in our model system) magnetic LRO can be established, where the $q=0$ symmetry is favorable if $J_{\perp}$ is of moderate strength. Thus, the observed $q=0$ magnetic order found in $\mathrm{Cs}_{2} \mathrm{Cu}_{3} \mathrm{SnF}_{12}$ and ascribed in Refs. 62 and [63] to anisotropy terms could also be attributed to the ILC without further anisotropy terms. The facilitation of the $\sqrt{3} \times \sqrt{3}$ magnetic long-range order found in the present paper for larger values of $\left|J_{\perp}\right|$ is related to a very small energy gain. In real compounds even very small additional terms in the relevant spin Hamiltonian such as further distance exchange couplings may therefore be more relevant.

\section{ACKNOWLEDGMENTS}

We thank H. Rosner, H. Tanaka, O. Janson and O. Derzhko for fruitful discussions.
[1] R. R. P. Singh and D. A. Huse, Phys. Rev. Lett. 68, 1766 (1992).

[2] C. Waldtmann H.-U. Everts, B. Bernu, C. Lhuillier, P. Sindzingre, P. Lecheminant, and L. Pierre, Eur. Phys. J. B 2, 501 (1998).

[3] S. Capponi, A. Läuchli, and M. Mambrini, Phys. Rev. B 70, 104424 (2004).

[4] R. R. P. Singh and D. A. Huse, Phys. Rev. B 76, 180407(R) (2007).

[5] G. Evenbly and G. Vidal, Phys. Rev. Lett. 104, 187203 (2010).

[6] S. Yan, D. A. Huse, and S. R. White, Science 332, 1173 (2011).

[7] H. Nakano and T. Sakai, J. Phys. Soc. Jpn. 80, 053704 (2011).

[8] A. M. Läuchli, J. Sudan, and E. S. Sørensen, Phys. Rev. B 83, 212401 (2011).

[9] Y. Iqbal, F. Becca, and D. Poilblanc, Phys. Rev. B 84, 020407(R) (2011).

[10] O. Götze, D.J.J. Farnell, R.F. Bishop, P.H.Y. Li, and J. Richter, Phys. Rev. B 84, 224428 (2011).

[11] S. Depenbrock, I. P. McCulloch, and U. Schollwöck, Phys. Rev. Lett. 109, 067201 (2012).

[12] Y. Iqbal, F. Becca, S. Sorella, D. Poilblanc, Phys. Rev. B 87, 060405(R) (2013).

[13] I. Rousochatzakis, R. Moessner, J. van den Brink, Phys. Rev. B 88, 195109 (2013).

[14] Z. Y. Xie, J. Chen, J. F. Yu, X. Kong, B. Normand, T. Xiang, Phys. Rev. X 4, 011025 (2014).

[15] I. Rousochatzakis, Y. Wan, O. Tchernyshyov, and F. Mila, Phys. Rev. B 90, 100406(R) (2014).

[16] W.-J. Hu, S.S. Gong, F. Becca, and D.N. Sheng, Phys. Rev. B 92, 2015(R) (2015).

[17] M. P. Zaletel and A. Vishwanath, Phys. Rev. Lett. 114, 077201 (2015).

[18] J. Oitmaa and R. R. P. Singh, Phys. Rev. B 93, 014424 (2016)

[19] O. Cépas and A. Ralko, Phys. Rev. B 84, 020413 (2011).

[20] T. Liu, W. Li, A. Weichselbaum, J. von Delft, and G. Su, Phys. Rev. B 91, 060403 (2015).

[21] H. J. Changlani and A. M. Läuchli, Phys. Rev. B 91, 100407 (2015).

[22] S. Nishimoto and M. Nakamura, Phys. Rev. B 92, 140412(R) (2015).

[23] W. Li, A. Weichselbaum, J. von Delft, and H.-H. Tu, Phys. Rev. B 91, 224414 (2015).

[24] P. Ghosh, A.K. Verma, and B. Kumar, Phys. Rev. B 93, 014427 (2016).

[25] Tao Liu, Wei Li, Gang Su, arXiv:1603.01935 1.

[26] O. Cepas, C. M. Fong, P. W. Leung, C. Lhuillier, Phys. Rev. B 78, 140405(R) (2008).

[27] I. Rousochatzakis, S. R. Manmana, A. M. Läuchli, B. Normand, and F. Mila, Phys. Rev. B 79, 214415 (2009).

[28] A. L. Chernyshev and M. E. Zhitomirsky, Phys. Rev. Lett. 113, 237202 (2014).

[29] Y.C. He and Y. Chen, Phys. Rev. Lett. 114, 037201 (2015)

[30] O. Götze and J. Richter, Phys. Rev. B 91, 104402 (2015).

[31] W. Zhu, S. S. Gong, and D. N. Sheng, Phys. Rev. B 92, 014424 (2015). 
[32] K. Kumar, K. Sun, and E. Fradkin, Phys. Rev. B 92, 094433 (2015).

[33] A. L. Chernyshev and M. E. Zhitomirsky, Phys. Rev. B 92, 144415 (2015).

[34] K. Essafi, O. Benton, and L.D.C. Jaubert, Nat. Commun. 7, 10297 (2016)

[35] J.-C. Domenge, P. Sindzingre, C. Lhuillier, and L. Pierre, Phys. Rev. B 72, 024433 (2005).

[36] O. Janson, J. Richter, and H. Rosner, Phys. Rev. Lett. 101, 106403 (2008).

[37] R.F. Bishop, P.H.Y. Li, D.J.J. Farnell, and C.E. Campbell, Phys. Rev. B 82, 104406 (2010).

[38] T. Tay and O. I. Motrunich, Phys. Rev. B 84, 020404(R) (2011).

[39] P.H.Y. Li, R.F. Bishop, C.E. Campbell, D.J.J. Farnell, Phys. Rev. B 86, 214403 (2012).

[40] H.-C. Jiang, Z. Wang, and L. Balents, Nature Physics 8, 902 (2012).

[41] B. Bauer, L. Cincio, B.P. Keller, M. Dolfi, G. Vidal, S. Trebst, and A.W.W. Ludwig, Nature Communications 5, 5137 (2014).

[42] R. Suttner, C. Platt, J. Reuther, and R. Thomale, Phys. Rev. B 89, 020408(R) (2014).

[43] W.J. Hu, W. Zhu, Y. Zhang, S.S. Gong, F. Becca, and D.N. Sheng Phys. Rev. B 91, 041124(R) (2015)

[44] S.S. Gong, W. Zhu, L. Balents, and D. N. Sheng Phys. Rev. B 91, 075112 (2015).

[45] F. Kolley, S. Depenbrock, I. P. McCulloch, U. Schollwöck, and V. Alba, Phys. Rev. B 91, 104418 (2015).

[46] S. Bieri, L. Messio, B. Bernu, and C. Lhuillier, Phys. Rev. B 92, 060407(R) (2015).

[47] A. Wietek, A.Sterdyniak, and A. M. Laeuchli, Phys. Rev. B 92, 125122 (2015).

[48] Y. Iqbal, H. O. Jeschke, J. Reuther, R. Valenti, I.I. Mazin, M. Greiter, and R. Thomale, Phys. Rev. B 92, 220404 (2015).

[49] P. Mendels, F. Bert, M. A. de Vries, A. Olariu, A. Harrison, F. Duc, J. C. Trombe, J. S. Lord, A. Amato, and C. Baines, Phys. Rev. Lett. 98, 077204 (2007).

[50] J. S. Helton, K. Matan, M. P. Shores, E. A. Nytko, B. M. Bartlett, Y. Yoshida, Y. Takano, A. Suslov, Y. Qiu, J.-H. Chung, D. G. Nocera, and Y. S. Lee, Phys. Rev. Lett. 98, 107204 (2007).

[51] M. A. de Vries, J. R. Stewart, P. P. Deen, J. Piatek, G. N. Nilsen, H. M. Ronnow, and A. Harrison, Phys. Rev. Lett. 103, 237201 (2009).

[52] D. Wulferding, P. Lemmens, P. Scheib, J. Röder, P. Mendels, S. Chu, T. Han, and Y. S. Lee, Phys. Rev. B 82, 144412 (2010).

[53] T. H. Han, J. S. Helton, S. Chu, D. G. Nocera, J. A. Rodriguez-Rivera, C. Broholm, and Y. S. Lee, Nature (London) 492, 406 (2012).

[54] B. Fak, E. Kermarrec, L. Messio, B. Bernu, C. Lhuillier, F. Bert, P. Mendels, B. Koteswararao, F. Bouquet, J. Ollivier, A. D. Hillier, A. Amato, R. H. Colman, and A. S. Wills, Phys. Rev. Lett. 109, 037208 (2012).

[55] E. Kermarrec, A. Zorko, F. Bert, R. H. Colman, B. Koteswararao, F. Bouquet, P. Bonville, A. Hillier, A. Amato, J. van Tol, A. Ozarowski, A. S. Wills, and P. Mendels, Phys. Rev. B 90, 205103 (2014).

[56] B. Bernu, C. Lhuillier, E. Kermarrec, F. Bert, P. Mendels, R. H. Colman, and A. S. Wills, Phys. Rev. B 87, 155107 (2013).
[57] H. O. Jeschke, F. Salvat-Pujol, and R. Valenti, Phys. Rev. B 88, 075106 (2013).

[58] H. Ishikawa, Y. Okamoto, and Z. Hiroi, J. Phys. Soc. Jpn. 82, 063710 (2013).

[59] T.-H. Han, J. Singleton, J. A. Schlueter, Phys. Rev. Lett. 113, 227203 (2014).

[60] H. O. Jeschke, F. Salvat-Pujol, E. Gati, N. H. Hoang, B. Wolf, M. Lang, J. A. Schlueter, and R. Valenti, Phys. Rev. B 92, 094417 (2015).

[61] T. Ono, K. Morita, M. Yano, H. Tanaka, K. Fujii, H. Uekusa, Y. Narumi, and K. Kindo, Phys. Rev. B 79, 174407 (2009).

[62] T. Ono, K. Matan, Y. Nambu, T. J. Sato, K. Katayama, S. Hirata, and H. Tanaka, J. Phys. Soc. Jpn. 83, 043701 (2014).

[63] K. Katayama, N. Kurita, and H. Tanaka, Phys. Rev. B 91, 214429 (2015).

[64] D. Boldrin, B. Fak, M. Enderle, S. Bieri, J. Ollivier, S. Rols, P. Manuel, and A. S. Wills, Phys. Rev. B 91, 220408(R) (2015).

[65] D. Schmalfuß, J. Richter and D. Ihle, Phys. Rev. B 70 $184412(2004)$

[66] V. Yu. Irkhin and A. A. Katanin, Phys. Rev. B 55, 12318 (1997).

[67] C. Yasuda, S. Todo, K. Hukushima, F. Alet, M. Keller, M. Troyer, and H. Takayama, Phys. Rev. Lett. 94, 217201 (2005).

[68] R.F. Bishop, in Microscopic Quantum Many-Body Theories and Their Applications, edited by J. Navarro and A. Polls, Lecture Notes in Physics 510 (Springer, Berlin, 1998), p.1.

[69] D.J.J. Farnell and R.F. Bishop, in Quantum Magnetism, Lecture Notes in Physics 645, 307 (2004)

[70] D. Schmalfuß, R. Darradi, J. Richter, J. Schulenburg, and D. Ihle, Phys. Rev. Lett. 97, 157201 (2006).

[71] R. Darradi, O. Derzhko, R. Zinke, J. Schulenburg, S.E. Krüger and J. Richter, Phys. Rev. B 78, 214415 (2008).

[72] R. Zinke, J. Schulenburg, and J. Richter, Eur. Phys. J. B 61, 147 (2008).

[73] D.J.J. Farnell, R. Zinke, J. Schulenburg, and J. Richter, J. Phys.: Cond. Matter 21, 406002 (2009).

[74] J. Richter, R. Darradi, J. Schulenburg, D.J.J. Farnell, and H. Rosner, Phys. Rev. B 81, 174429 (2010).

[75] D.J.J. Farnell, R.F. Bishop, P.H.Y. Li, J. Richter, and C.E. Campbell, Phys. Rev. B 84, 012403 (2011).

[76] D.J.J. Farnell, O. Götze, J. Richter, R.F. Bishop, and Phys. Rev. B 89, 184407 (2014).

[77] R. F. Bishop, P. H. Y. Li, and C. E. Campbell, Phys. Rev. B 89, 214413 (2014).

[78] J.-J. Jiang, Y.-J Liu, F. Tang, C.-H. Yang, and Y.-B. Sheng, Physica B: Cond. Mat. 463, 30 (2015).

[79] J. Richter, R. Zinke, D.J.J. Farnell, Eur. Phys. J. B 88, 2 (2015).

[80] P. H. Y. Li, R. F. Bishop, and C. E. Campbell, Phys. Rev. B 91, 014426 (2015).

[81] R.F. Bishop, D.J.J. Farnell, S.E. Krüger, J.B. Parkinson, J. Richter, and C. Zeng, J. Phys.: Condens. Matter 12, 6887 (2000).

[82] M. Roger and J.H. Hetherington, Phys. Rev. B 41, 200 (1990).

[83] R.F. Bishop, J.B. Parkinson, and Y. Xian, Phys. Rev. B 43, R13782 (1991); Phys. Rev. B 44, 9425 (1991).

[84] C. Zeng, D.J.J. Farnell, and R.F. Bishop, J. Stat. Phys. 90, 327 (1998). 
[85] A. B. Harris, C. Kallin and A. J. Berlinsky, Phys. Rev. B 45, 2899 (1992).

[86] S. Sachdev, Phys. Rev. B 45, 12377 (1992).

[87] A. Chubukov, Phys. Rev. Lett. 69, 832 (1992).

[88] C. L. Henley and E. P. Chan, J. Magn. Magn. Mater. 140-144, 1693 (1995).

[89] For the numerical calculation we use the program package 'The crystallographic CCM' (D.J.J. Farnell and J. Schulenburg).

[90] S.-S. Gong, W. Zhu, D.N. Sheng, O.I. Motrunich, M.P.A. Fisher, Phys. Rev. Lett. 113, 027201 (2014).

[91] M. Holt, O. P. Sushkov, D. Stanek, and G. S. Uhrig, Phys. Rev. B 83, 144528 (2011).

[92] Z. Fan and Q.-L. Jie, Phys. Rev. B 89, 054418 (2014).

[93] D. J. J. Farnell and R. F. Bishop, Int. J. Mod. Phys. B 22, 3369 (2008).
[94] A.F. Barabanov and V.M. Berezovskii, J. Phys. Soc. Jpn. 63, 3974 (1994); Phys. Lett. A 186, 175 (1994); Zh. Eksp. Teor. Fiz. 106, 1156 (1994) (JETP 79, 627 (1994).

[95] L. Siurakshina, D. Ihle, and R. Hayn, Phys. Rev. B 64, 104406 (2001).

[96] M. Härtel et al., Phys. Rev. B 87, 054412 (2013).

[97] B.H. Bernhard, B. Canals, and C. Lacroix, Phys. Rev. B 66, 104424 (2002).

[98] O. Janson, DFT based microscopic magnetic modeling for low-dimensional spin systems, Ph.D. thesis, Technische Universität Dresden (2012)

[99] D. Guterding, R. Valenti, and H. O. Jeschke, arXiv:1605.08162 